\title{
Optimization of Asteroid Capture Missions using Earth Resonant Encounters
}

\author{
Rita Neves and Dr. Joan Pau Sánchez
}

\begin{abstract}
This paper describes a robust methodology to design Earth-resonant asteroid capture trajectories leading to Libration Point Orbits (LPOs). These trajectories consider two impulsive manoeuvres; one occurring before the first Earth encounter and a final one that inserts the asteroid into a stable hyperbolic manifold trajectory leading to an LPO of the Sun-Earth system. The first manoeuvre is key to exploit the chaotic perturbative effects of the Earth and obtain important reductions on the cost of inserting the asteroid into a manifold trajectory. The perturbative effects caused by the Earth are here modelled by means of a Keplerian Map approximation, and these are posteriorly compared with the dynamics of the Circular Restricted Three-Body Problem. Savings in the order of $50 \%$ of total $\Delta v$ are computed for four different asteroids.
\end{abstract}

Key words: Asteroid Capture; Earth-Resonant Encounters; Trajectory Optimization; Libration Point Orbits

\section{Introduction}

Asteroid capture and retrieval missions have been getting the attention of the scientific community for some years. There are thousands of asteroids in orbits relatively close to the Earth and new ones are discovered often; as of January 2017, there are over 15000 observed near-Earth asteroids (NEA), from which 5\% were only reported the year before..$^{5}$ The characteristics of most NEA are still unknown,

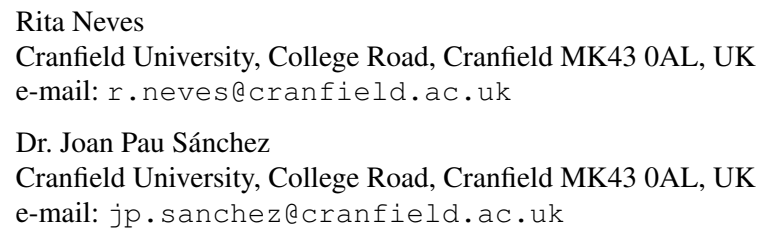


from size to material composition. As such, these bodies are considered very interesting targets for investigation. From data collection, to technology demonstrations or in-situ resource utilization, there are many scientific operations that can be undertaken, which presents an opportunity for challenging mission scenarios.

Asteroid capture missions are characterized by the rendezvous of a spacecraft with an asteroid and moving it to an orbit in the vicinity of the Earth. The spacecraft is utilised to modify the celestial body's trajectory in such a way as to make it enter the target orbit; the utilization of Solar Electric Propulsion (SEP) is one possible technology for this endeavour, ${ }^{9}$ although some others have been studied.

This work proposes to minimise the total fuel consumption, here regarded as $\Delta v_{C}$, of capturing an asteroid into a Libration Point Orbit (LPO). For this objective to be achieved, a manoeuvre that takes the asteroid from its nominal orbit to the destination has to be performed. This is the case studied by Yárnoz et al., ${ }^{11}$ which considers a single $\Delta v$ change that alters the asteroid's orbit to the one of the invariant manifold leading to the LPO, creating a database for Easily Retrievable Asteroids (EROs) by noting the capture $\Delta v$ of several bodies.

This work intends to exploit the chaotic nature of our Solar System and its numerous gravitational perturbations to find low-energy trajectories that lead to the capture of NEA into LPO. In this way, a different approach is proposed: the application of an initial manoeuvre $\Delta v_{M}$ for an optimal passage near the Earth, which is thereafter referred as an Earth-resonant encounter, and the final $\Delta v_{I}$ insertion into the LPO. It is proposed that the initial manoeuvre $\Delta v_{M}$ can be optimized in such a way that the resonant encounter with the Earth impacts the asteroid's orbital elements optimally, so that the total cost of the trajectory is lower than for a direct capture.

In order to model an asteroid's motion, the Keplerian Map (KM) equations are used. This is a perturbative model that allows for the simulation of Earth's gravitational influence on the body's orbit around the Sun, while being less computationally expensive than higher fidelity models such as the Circular Restricted Three-Body Problem (CR3BP). Given that the number of asteroids to be considered for capture is very high, utilizing this model is a way to decrease computational time. This is essential in space mission design, where several variables may have to be taken into account and, thus, extensive search spaces must be explored.

The present paper is organized as follows. Section 2 makes an overview on manifold theory and LPO, as well as detailing the dynamical models used for the presented asteroid capture trajectories, namely the KM. Section 3 analyses the full trajectory design and explains the procedure to obtain the best solution and its refinement with a higher order model. Section 4 reports the results for four different asteroids, studying the savings in $\Delta v_{C}$ and the impact on the capture's time of flight. Finally, Section 5 evaluates the implications of these developments and highlights some points that may benefit from further work. 


\section{Near-Earth Asteroids}

NEA have been so far classified according to their orbits and divided into four main categories: Amors, Apollos, Atens and Atiras. Amor asteroids stay always outside Earth's orbit and never cross it; Apollos and Atens cross Earth's orbit, but the former still have a wider orbit than the planet, while the latter are characterized for staying longer inside Earth's orbit and having smaller semi-major axes; Atiras remain confined inside Earth's orbit throughout their motion. These categories are depicted in Figure 1.

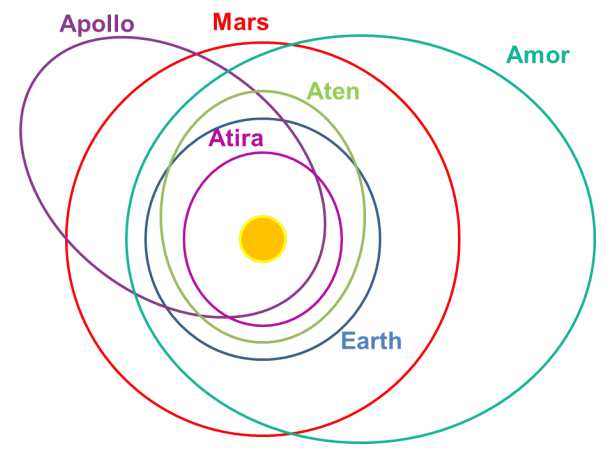

Fig. 1: Orbits of different NEA categories in the Solar System

The purpose of this work is to develop low-energy trajectories, using Earth-resonant encounters, that lead a NEA to an LPO. The latter are not the only near-Earth orbits that can be used for asteroid capture, but the asteroid population that can be cheaply moved into such an orbit may not be the same as into others (such as Distant Retrograde Orbits or DRO), as reported by Sánchez \& Yárnoz, ${ }^{8}$ making these interesting targets for investigation.

\subsection{LPO and Invariant Manifolds}

The Libration or Lagrangian points are positions in space where an object of negligible mass, affected by the gravitational interactions between two larger bodies (the primary and the secondary), can maintain a stationary position. These points are generally represented in a synodic reference frame, in which the primaries appear to be static while the third body rotates around them. They are very attractive for a great number of missions, namely to hold telescopes or other observation-type spacecraft, since the fuel consumption required to perform station-keeping is very low. 
Several types of periodic orbits can be found around these points, from which we highlight three: Horizontal Lyapunov orbits, which are in the ecliptic plane, Vertical Lyapunov orbits, that are horizontally symmetric and shaped like a figure-eight, and Halo orbits, which bifurcate from the Horizontal Lyapunov orbit family; these can be seen on Figure 2. An infinite number of quasi-periodic orbits can also be found, divided into two families: Lissajous around the Vertical Lyapunov orbits, and the Quasi-Halos around the Halo orbits. ${ }^{1}$

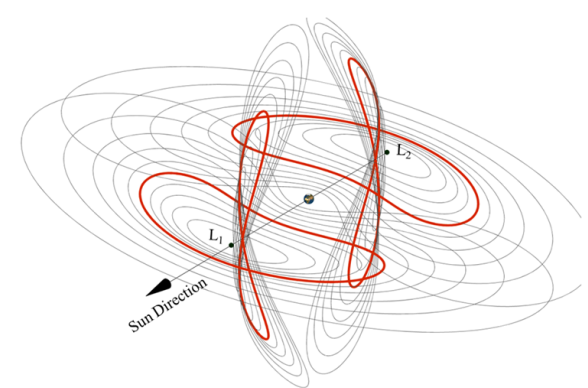

(a) Planar and Vertical Lyapunov orbits

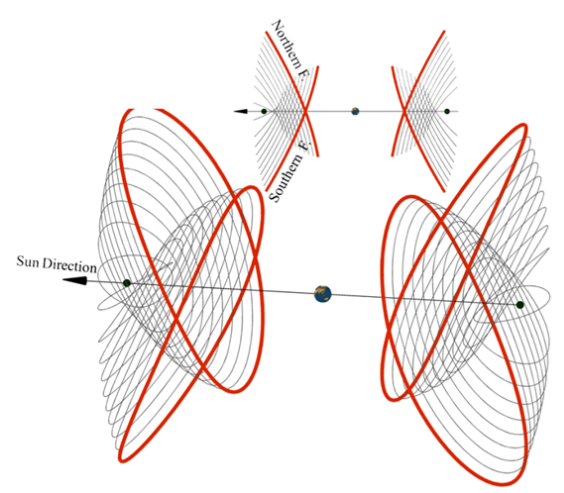

(b) Northern and southern families of halo orbits

Fig. 2: Libration Point Orbits associated with the Sun-Earth $L_{1}$ and $L_{2}$ points ${ }^{8}$

Hyperbolic invariant manifolds, dynamical structures composed of countless orbits, are connected to the LPOs. ${ }^{2}$ Mathematically, these are defined as sets of points in the system's phase space that tend toward a given limit as time tends to plus or minus infinity; they exist for a range of energies and form a series of 'tubes' connecting different regions around the primaries. These invariant manifold tubes can be used to explore new spacecraft trajectories with interesting characteristics: by moving one body to an invariant manifold orbit connected to an LPO, it will arrive there without any further manoeuvring.

\subsection{The Keplerian Map}

Considering that the asteroid encounters the Earth at some point along its trajectory, it is necessary to take into account its perturbative influence on the mission design. Since the considered asteroids move outside Hill sphere, it is infeasible to use a patched-conics method; therefore, we resort to the KM, a perturbation model for the motion of an object orbiting a central body. 
The KM influence is factored in using a first-order approximation of Picard's iteration on Lagrange's planetary equations. Its equations can be used to calculate the changes in orbital elements caused by the perturbing object, which are computed at each periapsis passage of the body, and then added to the previously known orbital elements. ${ }^{4}$ In this way, the action of the KM can be represented by the mapping $\mathscr{K}$ :

$$
\mathscr{K}:\{a, e, i, \omega \mid \alpha\} \mapsto\{\Delta a, \Delta e, \Delta i, \Delta \omega \mid \alpha\}
$$

The parameter $\alpha$ accounts for the phasing of the perturbed body with the one provoking the disturbance: in a synodic reference frame with the Sun (central body) and the Earth (perturbing body) as primaries, it is the angle in between the Sun-Earth line and the projection of the Sun-asteroid line in the ecliptic plane. Since the KM is only computed at $\alpha$ values in which a periapsis passage occurs, these are uniquely named $\alpha_{P}$. Considering the asteroid's movement, the value of $\Delta \alpha_{P}$ has also to be updated to represent the following periapsis passage, using this equation:

$$
\alpha_{P n+1}=\alpha_{P n}+2 \pi\left|\sqrt{\frac{a_{n+1}^{3}}{1-\mu}}-1\right|
$$

in which $n$ indicates in which time step the computation is being made, $a$ represents the asteroid's semi-major axis and $\mu$ is the normalized gravitational parameter of the system.

On Figure 3, we can observe the movement of asteroid 2016RD34 in the synodic reference frame; polar axes were juxtaposed to these, showing the range of $\alpha$. One of the periapsis passages happening during the Earth-resonant encounter is highlighted, revealing $\alpha_{P}=4.3^{\circ}$.

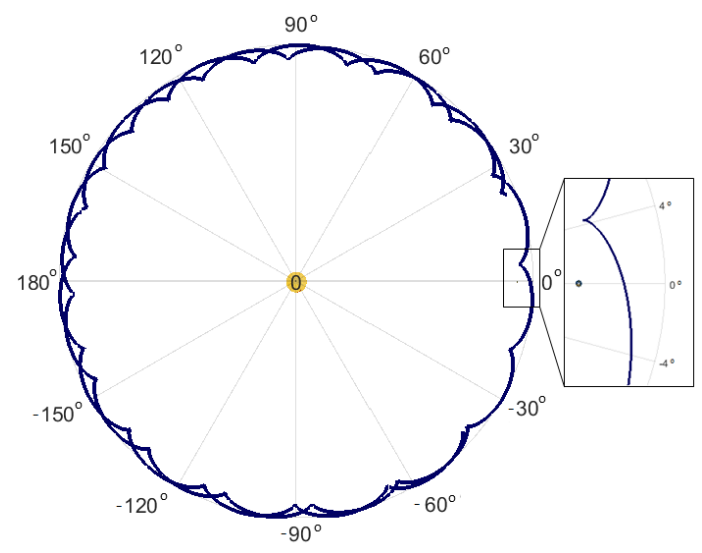

Fig. 3: Phasing $\alpha_{P}$ for an Earth-resonant encounter for asteroid 2016RD34 
An interesting application of the KM is the kick-map, a visual representation of the orbital elements changes as a function of the object's phasing with the perturbing body. As an example, Figure 4 shows the semi-major axis change undergone by asteroid 2016RD34 depending on the angle $\alpha_{P}$; this is, then, the kick-map that matches the movement shown in Figure 3. In this way, the value of $\Delta a$ corresponding to $\alpha_{P}=4.3^{\circ}$ can be simply taken from this plot—on Figure 4, it is represented by the crossing of dotted lines.

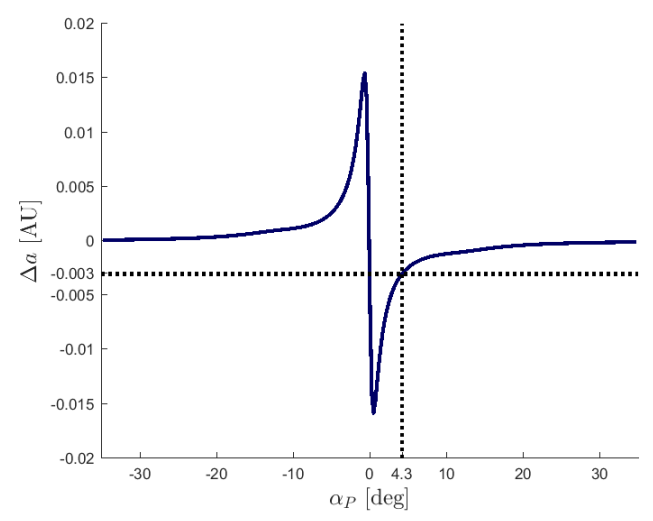

Fig. 4: Kick-map: change in $\Delta a$ with $\alpha$ for a resonant encounter of asteroid 2016RD34

The study of the kick-map also allows for the assertion that, for specific values of $\alpha_{P}$, the orbital element change can be quite significant, while for others the perturbing body's influence is barely felt.

\section{Trajectory Design}

The proposed trajectory consists on manoeuvring the asteroid for an optimal resonant encounter with the Earth and posterior capture into an LPO. Therefore, it can be divided into three distinct sections, highlighted in Figure 5. The first section, Phase A, starts when the asteroid is at the periapsis, right outside the perturbative region of influence of the Earth; at this point, the asteroid's velocity is changed by $\Delta v_{M}$, altering its path. The second section, Phase B, corresponds to the resonant encounter with the Earth, in which the asteroid is affected by its perturbation. This region was defined by $|\alpha|=\frac{\pi}{8}+\frac{\Delta \alpha}{2}$, which delimits a sufficiently large zone to encompass all $\alpha_{P}$ in which the object's motion is noticeably perturbed. The third section, Phase $\mathrm{C}$, ends at the insertion of the asteroid into an invariant manifold connected to an LPO 
by performing a manoeuvre of cost $\Delta v_{I}$. The final capture $\Delta v_{C}$ is the added total of the two different manoeuvres, $\Delta v_{M}$ and $\Delta v_{I}$.

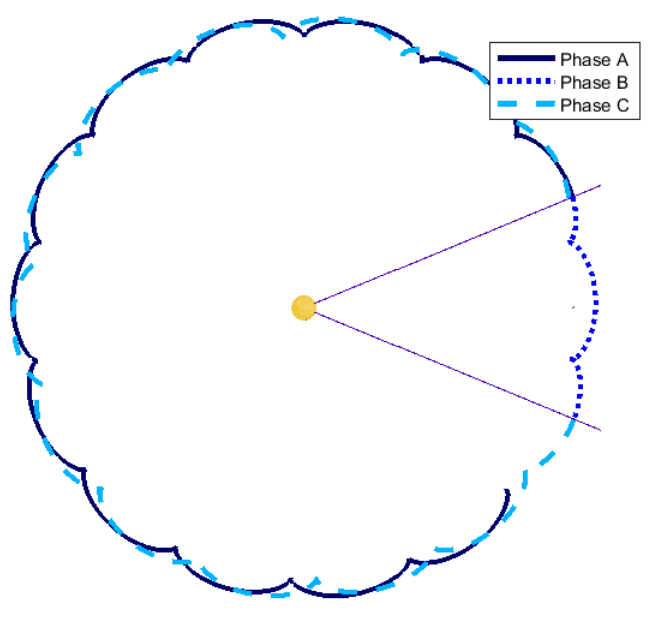

Fig. 5: Phases of the capture trajectory with an Earth-resonant encounter

The asteroid's motion during Phases A and C is Keplerian around the Sun; its path is only altered by $\Delta v_{M}$ on the former case. However, due to the close proximity with the Earth, Phase B has the object in a three-body configuration, where its movement is modelled with the KM. One simplification must be mentioned: although $\Delta v_{M}$ will cause a change in the orbital elements before the Earth-resonant encounter, it is so small that makes no difference in the application of the KM model. In this way, the mapping shown on Equation 1 was performed with the original set of orbital parameters, regardless of the application of $\Delta v_{M}$.

\subsubsection{Initial Filtering}

In order to assess which asteroids show reductions in capture cost by the implementation of this trajectory, a list of all discovered NEA orbital elements was collected from the Minor Planet Center's database. Considering over 15000 candidates, pruning had to be performed for this study to be feasible.

For this purpose, a filter that computes an estimate of the insertion cost $\Delta v_{I}$ in the LPO, based on a direct capture using a bi-impulsive manoeuvre, was designed. This was first described by Sánchez et. $\mathrm{al}^{6}$ and later expanded. ${ }^{8}$ It has proved to be a good lower threshold of the real capture manoeuvre and, as such, is used in the 
current paper to estimate $\Delta v_{I}$ for the computed trajectories. For completeness, this section includes a brief summary of how the filter works.

The bi-impulsive manoeuvre considers one burn on the perihelion and one on the aphelion, in which only one of the two is responsible for an inclination correction, and both include a semi-major axis change. This is described by Equation 3:

$$
\Delta v_{I}=\sqrt{\Delta v_{a 1}^{2}+\Delta v_{i 1}^{2}}+\sqrt{\Delta v_{a 2}^{2}+\Delta v_{i 2}^{2}}
$$

in which $\Delta v_{a}$ is the classical change in semi-major axis manoeuvre, whereas $\Delta v_{i}$ is the inclination change.

Thus, there are four computed values for $\Delta v_{I}$, depending on whether the perihelion or aphelion burn is the first and which of them will include the inclination correction; the lowest value out of these will be the filter output.

The filter application allowed, using an established ceiling of $1.2 \mathrm{~km} / \mathrm{s}$, to restrict our search to 61 asteroids. Posteriorly, the ephemerides from this reduced list were taken from the Horizons JPL database; ${ }^{3}$ the time period for data collection was from 2020 to 2100 . One synodic period, out of all comprised in this time span, was chosen: the one with highest optimization sensitivity, corresponding to the the one where the orbital elements suffer the greatest change as caused by the Earth.

\subsubsection{Grid Search}

In Figure 6, we observe the application of $\Delta v_{M}$ changes the asteroid's movement and encounter with the Earth, as opposed to its original path. It is important to denote that, for all the figures in this paper, the Earth is not in scale, but was plotted instead with the radius of Hill sphere. We can discern how a small change of the asteroids' orbital elements leads to a different encounter; therefore, our purpose is to develop a trajectory that leads to the cheapest capture possible by exploiting this effect. In order to achieve this, a grid search was performed for the $\Delta v_{M}$ corresponding to the lowest $\Delta v_{C}$.

As mentioned in Section 3, the KM is computed only for the initial asteroid's elements; as such, the orbital element changes will repeat themselves after the asteroid's orbit is moved forwards or backwards one epicycle, since $\alpha_{P}$ values will be the same. Thus, the analysis is restricted to $\Delta v_{M}$ inside limits that correspond to the asteroid moving backwards or forwards one epicycle. These are easily computed resorting to Gauss' form of the variational equations, ${ }^{6}$ as following:

$$
\Delta v_{M}=\frac{\mu_{S u n} \Delta a}{2 a^{2} v_{P}}
$$

where $\mu_{\text {Sun }}$ is the Sun's gravitational constant, $v_{P}$ is the velocity at the periapsis and $\Delta a$ represents the variation in initial semi-major axis corresponding to the addition of an extra epicycle to the asteroid's motion. The latter is computed using the equations: 


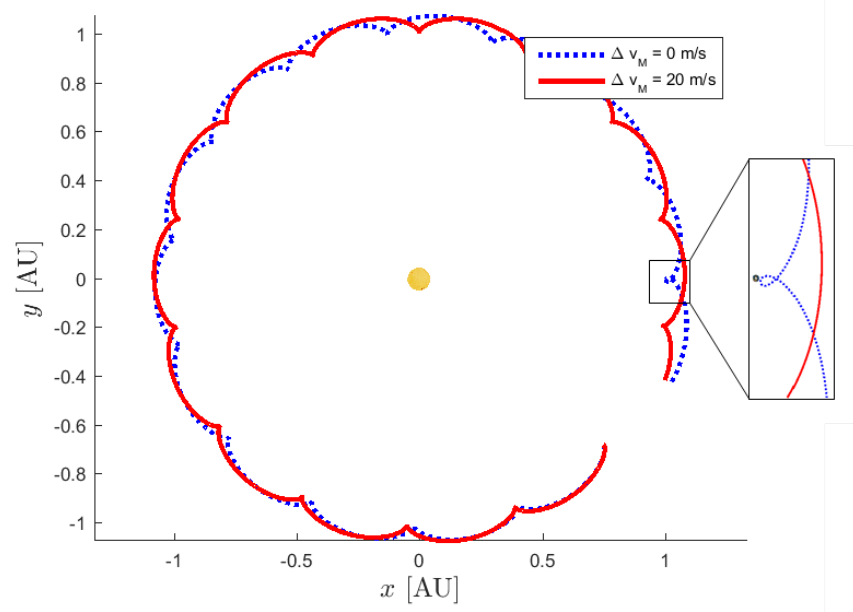

Fig. 6: Comparison of asteroid 2016RD34 trajectory with different initial values of $\Delta v_{M}$

$$
\Delta a=\frac{\sqrt{1-\mu} \Delta \alpha}{3 \pi n_{P} \sqrt{a}}
$$

where $\mu$ is the normalized gravitational constant of the system, $n_{P}$ is the number of periapsis passages occurring from the manoeuvre to the target point and $\Delta \alpha$ is the angular span of one epicycle:

$$
\Delta \alpha=2 \pi\left(\sqrt{\frac{a^{3}}{1-\mu}}-1\right)
$$

Once the limits are obtained, all the values of $\Delta v_{C}$ are computed for $\Delta v_{M}$ inside the established limits, with a step change of $0.2 \mathrm{~m} / \mathrm{s}$.

\subsubsection{Refinement}

After obtaining a solution using the method detailed in Subsection 3.0.2, the trajectory was refined with a higher fidelity model: namely, the CR3BP. This dynamical model is very well established for orbital motion, having been used countless times in mission analysis. ${ }^{10}$

For the purpose of obtaining a more refined solution, the CR3BP simulation has to be matched with the KM motion. Due to the higher sensitivity of the former, this may not be achieved by propagating the asteroid's motion using the orbital elements obtained after the $\Delta v_{M}$ manoeuvre. 
In general terms, the closer the asteroid is to the perturbing body, the greater its influence on it; as such, the object may undergo several periapsis passages in the region of the Earth's perturbation, but the one that will exert the most significant impact on its motion will be the one in which $\alpha_{P}$ is the closest to zero. Following this logic, in order to get a similar orbital change for the CR3BP as from the KM, the closest of its periapsis passages, $\alpha_{\text {closest }}$, should be the same as in the KM.

In order to target $\alpha_{\text {closest }}$, we employ a bisection method: this is implemented by defining upper and lower bounds to a trial $\Delta v_{M}$ and propagating the motion in the CR3BP using their mean value. Depending on whether the asteroid has surpassed or fallen behind $\alpha_{\text {closest }}$, the limits to the manoeuvre are changed and the consequent trial $\Delta v_{M}$ is altered accordingly.

On Figure 7, we can observe the evolution of the semi-major axis throughout time, for the entire trajectory, as depicted in Figure 5. Three plots can be distinguished: the propagation using the KM with the grid search solution $\left(\Delta v_{M}=-7.4 \mathrm{~m} / \mathrm{s}\right)$, with the CR3BP using the same manoeuvre and, finally, using the $\Delta v_{M}$ provided by the targeting method $\left(\Delta v_{M}=-6.6 \mathrm{~m} / \mathrm{s}\right)$. The latter option matches the first one much more accurately, reinforcing the choice to apply the targeting procedure.

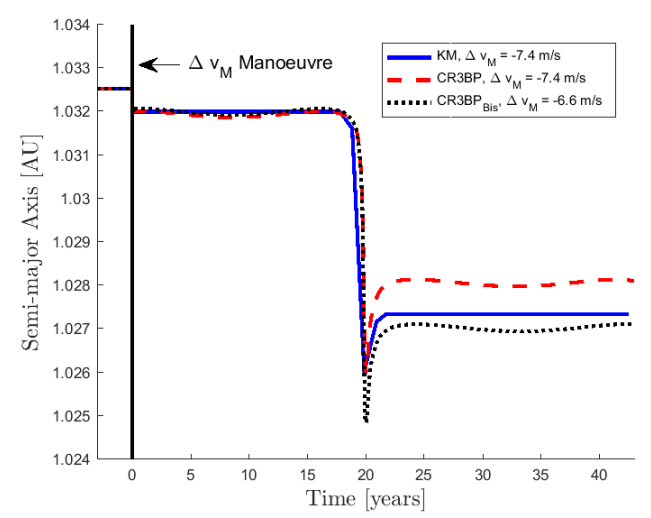

Fig. 7: Evolution of the semi-major axis of asteroid 2011BL45 throughout time

\section{Results and Discussion}

From the list of 61 asteroids that were filtered with a threshold of $\Delta v_{I}=1.2$ $\mathrm{km} / \mathrm{s}$, the ones presented in this paper are the four that benefit the most from the Earth-resonant encounter trajectory. In order to find these, three capture scenarios were compared and shown in Figure 8. Case 1 considers the cost of capturing an asteroid without any resonant encounter with the Earth; Case 2 examines a capture after the Earth-resonant encounter, but with no interference on the asteroid's 
path. Both these cases are used as benchmarks for comparison with our studied trajectory-the resonant capture with optimal manoeuvring $\Delta v_{M}$, depicted by Case 3.

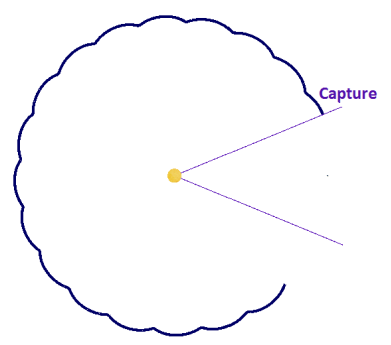

(a) Case 1: Direct Capture on (b) Case 2: Capture with zero First Synodic Period

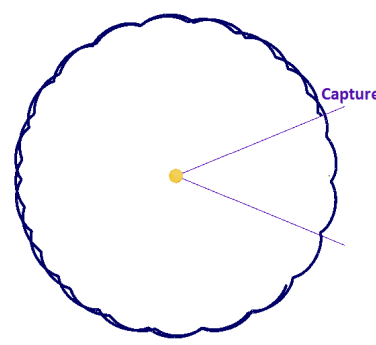

$\Delta v_{M}$, after Earth Resonance

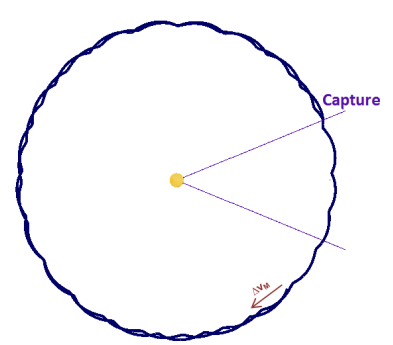

(c) Case 3: Capture with Optimal $\Delta v_{M}$, after Earth Resonance

Fig. 8: Capture Cases for asteroid 2011BL45

The asteroids selected were the ones presenting the highest savings in fuel consumption as compared to Cases 1 and 2. These results are presented on Table 1:

Table 1: Asteroid capture costs for each Case, in $[\mathrm{m} / \mathrm{s}]$

\begin{tabular}{lllllll}
\hline Asteroid & $\Delta v_{M}$ & $\Delta v_{\text {Case } 1}$ & $\Delta v_{\text {Case } 2}$ & $\Delta v_{\text {Case3 }}$ & $\Delta v_{C R 3 B P}$ & Fuel Reduction \\
\hline 2011BL45 & -7.4 & 88.0 & 116.1 & 48.4 & 45.1 & $45.0 \% \%^{1}$ \\
2010VQ98 & -2.6 & 255.3 & 470.4 & 112.2 & 120.1 & $56.1 \%{ }^{1}$ \\
2008UA202 & -13.6 & 307.3 & 307.6 & 189.0 & 214.5 & $38.5 \%^{1}$ \\
2016RD34 & -2.8 & 317.6 & 508.0 & 125.3 & 281.2 & $60.6 \%{ }^{1}$ \\
\hline
\end{tabular}

${ }^{1}$ Compared to Case 1

${ }^{2}$ Compared to Case 2

Two fuel reduction computations were obtained from comparing Case 3 to Cases 1 and 2; the value shown in Table 1 is the lowest of them, in order to highlight the asteroids in which Case 3 is clearly the most cost efficient. There are, however, asteroids which benefit greatly from a resonant trajectory, but in which the manoeuvre is not essential (i.e. asteroid 2011MD), which means that the $\Delta v$ of Cases 2 and 3 is similar - these were, consequently, left out of this discussion.

From Table 1, we observe that the obtained fuel reduction is very high, ranging from $38.5 \%$ to $60.6 \%$. The $\Delta v$ obtained for Cases 1 and 2 are very different; they correspond, in fact, to capture on two distinct synodic periods where substantial perturbation by the Earth was occurring regardless of any manoeuvre. However, in 
Table 2: Asteroid data and times of flight for capture manoeuvre, in years

\begin{tabular}{llllll}
\hline Asteroid & NEA Category & Capture LPO & Starting Date & TOF Case1 & TOF $_{\text {Case3 }}$ \\
\hline 2011BL45 & Amor & $\mathrm{VL}_{2}{ }^{1}$ & $19 / 08 / 2073$ & 17.6 & 42.4 \\
2010VQ98 & Apollo & $\mathrm{VL}_{2}{ }^{1}$ & $10 / 11 / 2063$ & 16.6 & 46.3 \\
2008UA202 & Apollo & $\mathrm{PL}_{2}{ }^{2}$ & $29 / 02 / 2020$ & 29.4 & 41.3 \\
2016RD34 & Amor & $\mathrm{VL}{ }^{1}$ & $18 / 10 / 2033$ & 12.9 & 35.4 \\
\hline
\end{tabular}

${ }^{1}$ Vertical Lyapunov in $L_{2}$

${ }^{2}$ Planar Lyapunov in $L_{2}$

the depicted cases, a very small $\Delta v_{M}$ corresponded to a significant decrease in $\Delta v$ for Case 3, as can be observed in Figure 9.

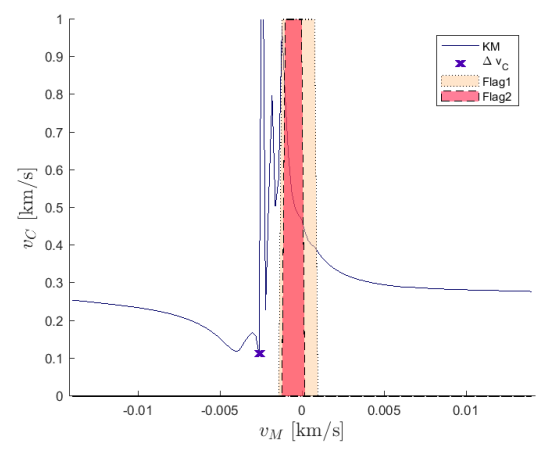

(a) 2010VQ98

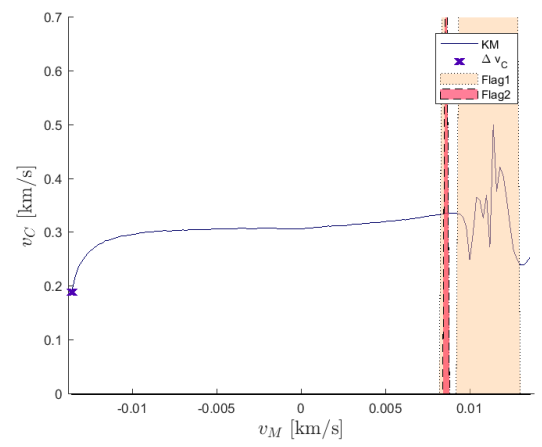

(c) 2008UA202

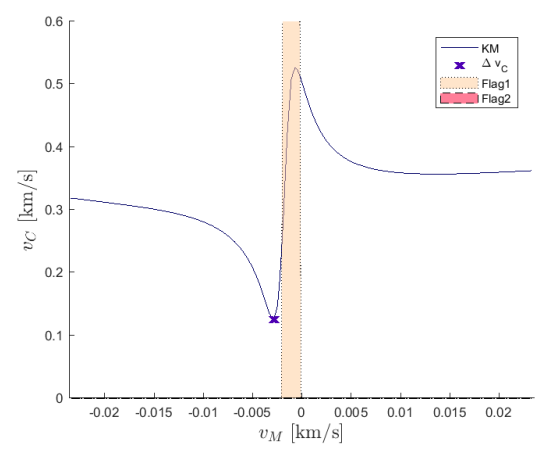

(b) 2016RD34

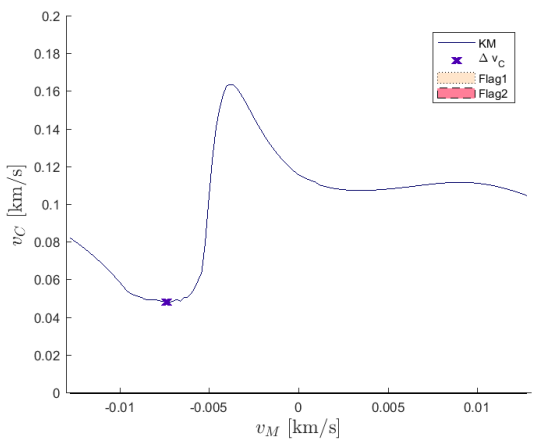

(d) 2011BL45

Fig. 9: Asteroid capture $\Delta v_{C}$ as a function of the initial manoeuvring $\Delta v_{M}$ 
In these figures, we can distinguish two coloured areas, Flag1 and Flag2. Flag1 is raised when the asteroid is moving inside the Hill sphere, whereas Flag2 appears when the perturbation is so strong that a transition occurs, meaning the asteroid's semi-major axis decreases from greater to smaller than one, or vice-versa; in both these cases, the KM should not be used to compute the body's motion, as previously mentioned in Section 2.2. It is possible to see how chaotic the plot behaviour becomes in these areas, corroborating this decision.

The refinement of $\Delta v_{\text {Case } 3}$ values with the CR3BP is shown on Table 1, represented by the parameter $\Delta v_{C R 3 B P}$. The targeting of $\alpha_{\text {closest }}$ using the bisection method converges quickly with great results: the error for the targeted angle reduces to less that $10^{-8}$ rad in a very short computational time- -this corresponds to a distance of about $80 \mathrm{~km}$ in the orbital motion.

For the first three cases, the values of $\Delta v_{C R 3 B P}$ appear very similar to $\Delta v_{\text {Case } 3}$; however, for asteroid 2016RD34, the difference is considerable. We conjecture that this has to do with the close proximity to the Hill radius of this solution, as we can see on Figure $9 \mathrm{~b}$. As such, for this specific case, we have allowed for a relaxation of the targeting error up to $10^{-3} \mathrm{rad}$ (about four times the previous distance) in order to check similar solutions. The obtained $\Delta v_{C R 3 B P}$ becomes $90 \mathrm{~m} / \mathrm{s}$, a fact that reiterates the increased sensitivity of the motion around Hill sphere and the need for cohesive establishment of the limits in which adequate solutions can be found, something that has been brought to attention by Sánchez et al. ${ }^{7}$

On Table 2, it is possible to see the categories these asteroids fall into, the target LPO and the times of flight of the capture trajectory with and without the resonant encounter. It is obvious that the savings in fuel cost are contrasted by the increased time of flight taken by the capture; since we are considering one extra synodic period, the trajectory takes roughly twice as long.

\section{Conclusions and Future Work}

The trajectories analysed show great promise in reducing $\Delta v$ costs of capturing asteroids into LPO. This is relevant in terms of mission design, since fuel consumption is one of the primary impactors of the cost of a space mission, and as such one of the main constraints limiting their boldness. However, the savings in fuel cost have to be weighted against the increased time of flight spent in the entire capture. This is a matter of trade-off analysis that can be performed for a specific mission design. Furthermore, a careful selection of the synodic period of the capture, by itself, is also valuable for cost reduction.

In summary, we have presented a new tool, supported by a dynamical model of motion of low computational cost, that proves to be very efficient in the design of optimal resonant encounters. This tool can be applied to the study of several interesting cost-saving trajectories in other planetary configurations and missions, such as Jovian moon tours. 
The KM is shown to behave very similarly to the CR3BP and to adequately model the complexity of low energy resonant motion in asteroids where there is no transition and its distance to the Earth is always greater than Hill radius. Further work should consider the analysis of the boundaries of the KM, meaning the exact limits where it stops being a good approximation to a higher fidelity model.

In regards to resonant capture trajectories, further work will focus on the entire optimization of the manoeuvres, without resorting to the filter described in Section 3.0.1; instead of admitting a bi-impulsive manoeuvre estimate, an optimized Lambert arc will be considered. Furthermore, the use of low-thrust systems in the computation of these trajectories will also be studied and compared to current chemical thrust solutions.

\section{References}

1. Gómez G, Koon WS, Lo MW, Marsden JE, Masdemont J, Ross SD (2004) Connecting Orbits and Invariant Manifolds in the Spatial Restricted Three-Body Problem. Nonlinearity 17(5)

2. Koon WS, Lo MW, Marsden JE, Ross SD (2000) Heteroclinic Connections Between Periodic Orbits and Resonance Transitions in Celestial Mechanics. Chaos: An Interdisciplinary Journal of Nonlinear Science 10:427-469

3. Park P, Chamberlin A (2017) Horizons JPL Asteroid Database. http: / / ssd. jpl . nasa . gov/horizons.cgi\#top Accessed: 2017-01-10

4. Ross SD, Scheeres DJ (2007) Multiple Gravity Assists, Capture, and Escape in the Restricted Three-Body Problem. SIAM Journal on Applied Dynamical Systems 6:576-596

5. Rudenko M (2017) Minor Planet Center Asteroid Database. http://www . minorplanetcenter. net/iau/TheIndex.html. Accessed: 2017-01-10

6. Sánchez JP, Alessi EM, Yárnoz DG, McInnes C (2013) Earth Resonant Gravity Assists for Asteroid Retrieval Missions. 64th International Astronautical Congress 201313

7. Sánchez JP, Colombo C, Alessi EM (2015) Semi-Analytical Perturbative Approaches to Third Body Resonant Trajectories. Proceedings of the International Astronautical Congress, IAC 7:5504-5515

8. Sánchez JP, Yárnoz DG (2016) Asteroid Retrieval Missions Enabled by Invariant Manifold Dynamics. Acta Astronautica

9. Strange N, Landau D, Lantoine G, Lam T, McGuire M, Burke L, Martini M, Dankanich J (2013) Overview of Mission Design for NASA Asteroid Redirect Robotic Mission Concept. $33^{\text {rd }}$ International Electric Propulsion Conference

10. Szebehely V (1969) Theory of Orbit. Academic Press

11. Yárnoz DG, Sánchez JP, McInnes CR (2013) Easily Retrievable Objects Among the NEO Population. Celestial Mechanics and Dynamical Astronomy 116:367-388 
2018-02-11

\section{Optimization of asteroid capture missions using Earth resonant encounters}

Neves, Rita

Springer

Rita Neves, and Joan Pau Sánchez. Optimization of asteroid capture missions using Earth resonant encounters. In: Vasile M., Minisci E., Summerer L., McGinty P. (eds) Stardust Final Conference on Asteroids and Space Debris, Volume 52, 2018 https://doi.org/10.1007/978-3-319-69956-1_1

Downloaded from Cranfield Library Services E-Repository 